\title{
Validity of an Interviewer-Administered Patient Health Questionnaire-9 to Screen for Depression in HIV-Infected Patients in Cameroon
}

\author{
Brian W. Pence, PhD MPH ${ }^{1,2}$, Bradley N. Gaynes, MD MPH ${ }^{3}$, Julius Atashili, MD PhD MPH ${ }^{4}$, \\ Julie K. O'Donnell, MPH ${ }^{5}$, Gladys Tayong, MD ${ }^{6}$, Dmitry Kats, MPH $^{5}$, Rachel Whetten, $\mathbf{M P H}^{2}$, \\ Kathryn Whetten, PhD MPH ${ }^{2,7}$, Alfred K. Njamnshi, MD MA ${ }^{8}$, and Peter M. Ndumbe, MD \\ $\mathrm{PhD}^{9}$ \\ ${ }^{1}$ Department of Community and Family Medicine, Duke University, Durham, NC, USA \\ ${ }^{2}$ Center for Health Policy and Inequalities Research, Duke Global Health Institute, Duke \\ University, Durham, NC, USA \\ ${ }^{3}$ Department of Psychiatry, University of North Carolina School of Medicine, Chapel Hill, NC, USA \\ ${ }^{4}$ Department of Public Health and Hygiene, University of Buea, Cameroon \\ ${ }^{5}$ Department of Epidemiology, Gillings School of Global Public Health, University of North \\ Carolina, Chapel Hill, NC, USA \\ ${ }^{6}$ Bamenda Regional Hospital, Bamenda, Cameroon \\ ${ }^{7}$ Sanford School of Public Policy, Duke University, Durham, NC, USA \\ ${ }^{8}$ Department of Internal Medicine (Neurology Unit), The University of Yaoundé I, Yaoundé, \\ Cameroon \\ ${ }^{9}$ Department of Biomedical Sciences, University of Buea, Cameroon; Department of Microbiology \\ and Immunology, University of Yaoundé I, Yaoundé, Cameroon
}

\section{Abstract}

\begin{abstract}
Background-In high-income countries, depression is prevalent in HIV patients and is associated with lower medication adherence and clinical outcomes. Emerging evidence from lowincome countries supports similar relationships. Yet little research has validated rapid depression screening tools integrated into routine HIV clinical care.
\end{abstract}

Methods-Using qualitative methods, we adapted the Patient Health Questionnaire-9 (PHQ-9) depression screening instrument for use with Cameroonian patients. We then conducted a cross-

\footnotetext{
(C) 2012 Elsevier B.V. All rights reserved

Corresponding author: Brian W. Pence, PhD MPH, Associate Professor, Department of Community and Family Medicine, Duke Global Health Institute, and Center for Health Policy and Inequalities Research, Box 90392, Duke University, 2812 Erwin Rd, Durham, NC 27705 (brian.pence@ duke.edu telephone: (919)-613-5443; fax: 919-613-5466)..

Publisher's Disclaimer: This is a PDF file of an unedited manuscript that has been accepted for publication. As a service to our customers we are providing this early version of the manuscript. The manuscript will undergo copyediting, typesetting, and review of the resulting proof before it is published in its final citable form. Please note that during the production process errors may be discovered which could affect the content, and all legal disclaimers that apply to the journal pertain.

Conflicts of interest: The authors state that no relevant conflicts of interest exist.

Author contributions: BWP, BNG, JA, KW, RW, PN obtained funding and designed the study. BWP, BNH, JA, GT, RW, PN oversaw training and data collection. BWP and JKO analyzed the data. BWP, BNG, JA, JKO drafted the manuscript. DK reviewed the literature. GT, DK, RW, KW, PN reviewed the manuscript for important intellectual content. All authors contributed to and have approved the final manuscript.
} 
sectional validity study comparing an interviewer-administered PHQ-9 to the reference standard Composite International Diagnostic Interview in 400 patients on antiretroviral therapy attending a regional HIV treatment center in Bamenda, Cameroon.

Results-The prevalence of major depressive disorder (MDD) in the past month was 3\% ( $\mathrm{n}=11$ cases). Using a standard cutoff score of $\geq 10$ as a positive depression screen, the PHQ-9 had estimated sensitivity of $27 \%$ (95\% confidence interval: 6-61\%) and specificity of 94\% (91-96\%), corresponding to positive and negative likelihood ratios of 4.5 and 0.8 . There was little evidence of variation in specificity by gender, number of HIV symptoms, or result of a dementia screen.

Limitations-The low prevalence of MDD yielded very imprecise sensitivity estimates. Although the PHQ-9 was developed as a self-administered tool, we assessed an intervieweradministered version due to the literacy level of the target population.

Conclusion-The PHQ-9 demonstrated high specificity but apparently low sensitivity for detecting MDD in this sample of HIV patients in Cameroon. Formative work to define the performance of proven screening tools in new settings remains important as research on mental health expands in low-income countries.

\section{Keywords}

HIV; depression; validation study; screening instruments; Africa

\section{Introduction}

In high-income nations, depression in HIV patients has received much attention as a highly prevalent comorbidity and a predictor of reduced antiretroviral (ARV) medication adherence, higher transmission risk behaviors, and poorer clinical outcomes. ${ }^{1-3}$ As access to HIV clinical care and ARVs expands in sub-Saharan Africa, ${ }^{4}$ increasing attention has been focused on the prevalence and consequences of depression for HIV-infected patients in these settings. ${ }^{3}$ Evidence to date suggests that in sub-Saharan Africa, as in other parts of the world, the prevalence of depression is higher in HIV patients than in the general population and that depression is associated with worse ARV adherence and HIV clinical outcomes. ${ }^{2,5}$

In order to respond to the potential negative impact of depression on HIV clinical care, HIV clinical settings require rapid, validated screening tools to identify patients with a likely depressive disorder. Brief depression screening tools such as the Patient Health Questionnaire-9 (PHQ-9) ${ }^{6}$ are becoming widely used in the United States and Europe, but these tools were generally developed for self-administration by a literate population. Few studies ${ }^{7}$ have been conducted in African countries validating such tools against reference standard diagnostic assessments, especially in low-literacy populations, or have considered their application in HIV-infected populations specifically. ${ }^{8}$

Accordingly, we undertook to validate the PHQ-9 as a depression screening tool compared to the internationally calibrated Composite International Diagnostic Interview (CIDI) at a regional hospital serving a predominantly poor and low-literacy HIV patient population in the Northwest Region of Cameroon. Cameroon is a country of approximately 20 million people in Central Africa ${ }^{9}$ with an estimated HIV prevalence rate of $5.3 \% .{ }^{10}$

\section{Methods}

\section{Ethical approvals}

This study was approved by the Cameroon National Ethics Committee (No. 111/CNE/SE/ 09), the University of North Carolina at Chapel Hill's Biomedical Institutional Review 
Board (\# 09-0852) and the Duke University Health System IRB (\# Pro00016937). The study also received administrative approval from the Ministry of Public Health in Cameroon.

\section{Sample}

We conducted a cross-sectional validation study to compare depression screening results from the PHQ-9 to a reference standard tool for depression diagnosis among HIV-positive patients on antiretroviral therapy (ART) and attending the Bamenda Regional Hospital AIDS Treatment Center (BRHATC) in Cameroon. The BRHATC is a health facility dedicated to the care of HIV-positive patients, the vast majority of whom are on ART. The center provides care to over 4,000 patients annually. Patients were eligible if they were HIVinfected, on antiretroviral therapy ART, were attending the BRHATC for any service (including counseling, clinical follow-up or drug refill), spoke English, were ages 18-55, and were willing to provide informed consent. We excluded patients $>55$ years because the present study was to lay the groundwork for a medication trial which would exclude patients $>55$ years for safety reasons. Patients who could not give consent or refused to give consent were excluded. Although more than 30 local languages exist, English is the language used for regular clinical care in the BRHATC and throughout the Northwest Region of Cameroon; more than $95 \%$ of clinic attendees can communicate effectively in English. Each participant could be eligible only once during the study period.

Our sampling goal was to recruit a study sample representative of HIV-positive patients receiving ART at BRHATC. In the absence of a daily patient register to provide a sampling frame, study staff approached each patient consecutively as the patients passed through a central point in the registration process (weight measurement) until a patient indicated willingness to participate in the study. The recruiting staff member then obtained written informed consent from the interested patient and completed the first part of data collection. The patient would then complete the second part of data collection with a second staff member while the recruiting staff member resumed approaching patients consecutively at registration.

\section{Instruments}

PHQ-9-The PHQ-9 is a widely used 9-item screener for depression that assesses the presence within the past two weeks of the 9 core symptoms of depression as specified by the DSM-IV. ${ }^{11}$ Each symptom is rated as being present none of the time, a few days, more than half the days, or nearly every day in the past two weeks. The PHQ-9 total score can range from $0-27$, with a score of 10 or above being considered in many settings as an indication of a likely depressive disorder.

In order to assess any needed adaptations of the wording of the PHQ-9 for use in our setting, we conducted four focus groups with hospital patients and family members from our study site. Groups were separated by gender and religion (Christian and non-Christian). Study staff trained in qualitative methods led the focus groups, which were audio-recorded and transcribed for content analysis. The analysis suggested minor wording changes to two of the questions and optional clarifications (that were read only if the original question was not understood) for six additional questions.

Reference standard: CIDI—Diagnoses of major depression were established with the reference standard Composite International Diagnostic Instrument (CIDI) from the World Health Organization. The CIDI is a lay-administered diagnostic instrument whose performance has been validated widely in multiple international settings, including HIV settings. ${ }^{12,13}$ The CIDI is a comprehensive, fully structured interview designed to be used by trained lay interviewers for the assessment of mental disorders in epidemiological and cross- 
cultural studies as well as for clinical and research purposes. We used the World Mental Health Survey Initiative Version of the CIDI (WMH-CIDI), which allows the assessment of mental disorders according to the definitions and criteria of ICD-10 and DSM-IV. The diagnostic section of the interview expands upon earlier versions of the CIDI by adding detailed questions about disorder severity, impairment, service use, and treatment, and has improved generalizability with increased involvement of less wealthy countries. This version has been successfully used in Sub-Saharan Africa (Nigeria). ${ }^{14}$

\section{Procedures}

After consent, participants completed the PHQ-9 with one study team member. Given the low level of literacy in the target population, the PHQ-9 was read to all participants and responses were recorded on the form by the study team member, who was trained in PHQ-9 administration. Previous studies have found comparable results from interviewer administration and self-administration of the PHQ-9. ${ }^{15}$ Participants also provided sociodemographic information, completed the International HIV Dementia Scale, ${ }^{16}$ and were asked whether in the past 6 months they had experienced each of 13 symptoms commonly associated with HIV infection: new or persistent headaches, fevers, oral pain, white patches in the mouth, rashes, nausea, trouble with eyes, sinus infection, numbness in the hands or feet, persistent cough, diarrhea, weight loss, or (for women only) abnormal vaginal discharge.

Participants then completed the Screening and Depression modules of the CIDI in an inperson interview with a second study team member who was blinded to the results of the PHQ-9 screening. The CIDI interviewer was a health care professional who received formal CIDI training from a WHO-certified trainer. The CIDI interviewer completed regular supervision and record review with a psychiatrist (BNG) to ensure consistence and accuracy of diagnoses.

\section{Measures}

Based on validation studies of the PHQ-9 in other settings, we defined a positive screen for depression as a PHQ-9 total score of $\geq 10 .^{7,17}$ In sensitivity analyses we also considered alternative cutoffs of $\geq 8$ and $\geq 12$.

Standard CIDI scoring methodology requires the following components for a lifetime major depressive episode: a period lasting at least two weeks characterized by at least 5 out of 9 core depressive symptoms, with at least one symptom being either depressed mood or anhedonia, and representing a change from previous functioning; the symptoms must have caused clinically significant distress or impairment in social, occupational, or other important areas of functioning; the symptoms must not be better explained by substance use or a general medical condition; and the symptoms must not be better explained by bereavement, or if secondary to bereavement, the episode either must last more than two months or be characterized by at least one of the following: marked functional impairment, morbid preoccupation with worthlessness, suicidal ideation, psychotic symptoms, or psychomotor retardation.

On the CIDI, participants reporting any lifetime major depressive episode (MDE) were asked if they had experienced any similar episodes in the past year, past 6 months, past month, and currently. Participants reporting an episode in the past year or more recently completed a standard depressive severity rating scale called the Quick Inventory of Depressive Symptoms (QIDS) ${ }^{4}$ that is embedded within the CIDI. The QIDS total score can range from $0-27$ and has standard categories that correspond to very severe (21-27), severe (16-20), moderate (11-15), mild (6-10), and no (0-5) depressive symptoms. Participants 
were classified as having a diagnosis of a MDE in the past year, past 6 months, and past month if they endorsed a depressive episode in those time frames and received a score of 11 or above on the QIDS.

The IHDS was scored according to standard methodology. ${ }^{16}$ Each of three tasks (motor speed - number of finger taps in 5 seconds; psychomotor speed - number of sequences of a pattern of hand movements in 10 seconds; memory-recall - number of words recalled) is scored on a $0-4$ scale, with higher scores indicating better functioning and a maximum possible score of 12 . A score of 10 or below is considered a positive screen for possible dementia.

\section{Statistical analysis}

Participant characteristics are described with proportions or with means and standard deviations (SD). To calculate test characteristics of the PHQ-9 relative to the CIDI, we ideally would have compared a positive PHQ-9 screen (which references the past two weeks) to CIDI diagnoses of current MDE. However, the overall prevalence of MDE in the sample was lower than anticipated and an initial examination of the prevalence of MDE in different time frames indicated that there were too few cases of current MDE to allow meaningful estimation of sensitivity. We therefore made the decision for the primary analysis to compare the PHQ-9 to CIDI diagnoses of MDE in the past month, as has been done in prior studies, ${ }^{18,19}$ recognizing that the time frames referenced by the two instruments would differ.

We calculated the sensitivity, specificity, likelihood ratio positive (LR+), and likelihood ratio negative (LR-) for this comparison. Since positive and negative predictive value (PPV and NPV) vary by prevalence, we present what the PPV and NPV for a range of different prevalence values for depression. We examined whether there was evidence that the test characteristics of the PHQ-9 differed in certain subgroups (gender, HIV physical symptoms, English proficiency, and dementia score). In secondary analyses, we considered different thresholds for the PHQ-9 $(8,10$, and 12) and different time frames for the CIDI (current versus past month).

\section{Results}

Between May and October, 2010, 461 patients were approached; 48 were not eligible (26 did not speak English well enough to complete study activities, and 22 were $>55$ years old) and 13 declined to participate (10 did not have time and 3 wished to consult their spouse before participating). Informed consent was provided by the remaining 400 participants. Participants had a median age of 41 years (interquartile range: $34-47$ years); $74 \%$ were female, 99\% were Christian (religious affiliations were indicated as Muslim by four participants and traditional by one participant), and $61 \%$ had completed only primary education (Table 1). The self-reported estimated median household daily expenditures were FCFA 700 (IQR 400-1400) (approximately US \$1 [\$1-\$3]). Participants endorsed a median of 5 HIV-related symptoms (IQR: 3-6). A majority of participants (83\%) met criteria for possible dementia. More than half of the subjects performed poorly on the memory subscale (54\% with a score 3 out of 4 ).

Of 398 individuals with a complete CIDI assessment, 11 (3\%) met diagnostic criteria for a major depressive episode in the past month (Table 2). Of these 11 cases, 3 had a positive PHQ-9 screen for depression at a cutoff of 10 or above (true positives) (Table 3). Of the 387 participants without a diagnosis of an MDE in the past month, 364 had a negative PHQ-9 screen. Thus the standard PHQ-9 cutoff of 10 or above had a sensitivity of $27 \%$ (95\% CI: 6- 
$61 \%$ ) and a specificity of $94 \%$ (95\% CI: $91-96 \%$ ) relative to an MDE diagnosis on the CIDI in the past month. These test characteristics correspond to a LR+ of 4.5 and a LR- of 0.8 .

In sensitivity analyses, we compared the standard PHQ-9 cutoff score of 10 to current MDE diagnoses at the time of interview. Four participants (1\%) met criteria for a current MDE. When considering current MDE, the sensitivity of the PHQ-9 was higher than when considering past-month MDE (50\%, 95\% CI: 7-93\%). Specificity was unchanged at $94 \%$ (95\% CI: 91-96\%).

Compared to the original time frame of MDE in the past month, use of a lower PHQ-9 cutoff of 8 yielded a slightly higher sensitivity and lower specificity, while use of a higher PHQ-9 cutoff of 12 yielded a slightly lower sensitivity and higher specificity (Table 3). Given the low number of cases and the resulting wide uncertainty around the sensitivity estimate, we do not present a full ROC curve, as ROC estimates would be imprecise.

As PPV and NPV are functions of prevalence as well as sensitivity and specificity, we illustrate in Figure 1 the PPV and NPV for the observed sensitivity and specificity over a range of possible prevalence values of MDE. At the MDE prevalence of 3\% observed in this population (indicated by the red vertical line), the PHQ-9 had a PPV of $12 \%(2-30 \%)$ and an NPV of 98\% (96-99\%). In a hypothetical population with a higher MDE prevalence of 20\%, the PPV of the PHQ-9 would improve to 53\% while the NPV would decline to $84 \%$.

The number of MDE cases was too small to assess whether the sensitivity of the PHQ-9 varied for different subgroups. There was little evidence of variation in specificity by gender, number of HIV symptoms endorsed, or outcome of the International HIV Dementia Scale assessment (Table 4).

\section{Discussion}

In this population of predominantly low-literacy HIV-infected individuals in Cameroon, an interviewer-administered PHQ-9 demonstrated high specificity but low sensitivity in identifying cases of major depressive disorder as measured by the gold standard Composite International Diagnostic Interview (CIDI). The estimate of PHQ-9 specificity at the standard cutoff of 10 or above was comparable with other published results from a range of settings. ${ }^{17}$ The estimate of sensitivity at the standard cutoff was lower than many previous studies, especially in primary care populations, ${ }^{17}$ but was comparable to some studies done in chronically ill medical populations ${ }^{20}$ and to a recent large validation study in the Netherlands that also used the CIDI as its gold standard. ${ }^{19}$ The sensitivity was imprecisely estimated because of the small number of MDD cases in this population. As expected, the PHQ-9 (which asks about symptoms in the past two weeks) demonstrated higher sensitivity in detecting MDD that was present at the time of interview than MDD diagnoses from any point in the past month, although conclusions are limited by the low prevalence of cases available to calculate sensitivity.

The prevalence of depression was somewhat lower in this sample than in other recently reported studies of HIV-infected individuals in sub-Saharan Africa, ${ }^{21}$ although the majority of prevalence estimates from the region have been based on screening instruments rather than diagnostic instruments. Studies in the region using diagnostic instruments have reported MDD prevalence estimates ranging from $2.7-34.9 \%, 2-28$ with most studies employing the Mini International Neuropsychiatric Interview. ${ }^{29}$ The WHO Neuropsychiatric AIDS Study from the early 1990s, which was the only published study we could identify which used the CIDI to measure depression among HIV-infected individuals in the region, reported prevalence estimates in Nairobi, Kenya, of 3.0\% and 5.5\% in asymptomatic and symptomatic HIV-infected patients, respectively, and prevalence estimates in Kinshasa, 
Democratic Republic of the Congo, of $0 \%$ and $4.4 \%$ in asymptomatic and symptomatic HIV-infected patients. ${ }^{12}$

Although several studies have considered the internal and construct validity of the PHQ-9 in sub-Saharan African populations, ${ }^{30,31}$ including in HIV patients, ${ }^{8}$ we identified only one formal validation study from Africa that reported sensitivity and specificity of the PHQ-9 relative to a reference standard diagnostic instrument. ${ }^{7}$ This validation study compared a self-administered PHQ-9 to the Mini International Neuropsychiatric Interview (MINI) among a highly educated sample of Nigerian university students and reported a sensitivity of $85 \%$ and specificity of $99 \%$. Although promising, the performance of the PHQ-9 and other depression screening instruments needs to be better understood in Cameroon and other lowincome countries.

This study's findings should be understood in light of its strengths and limitations. The generalizability of the present study is enhanced by the consecutive sampling approach, broad inclusion criteria, and recruitment from a large regional HIV/AIDS treatment center. The validity of our screening and diagnostic measures of depression was enhanced by adaptation of the PHQ-9 for use in Cameroon through focus group feedback, rigorous training of interviewers, regular review of diagnostic decisions by a supervising psychiatrist, and blinding of the CIDI interviewer to the results of the PHQ-9 screening. Due to the low level of literacy in the target population, we chose to have the PHQ-9 be intervieweradministered rather than self-administered. The PHQ-9 was developed as a self-administered tool and only a small number of studies have validated an interviewer-administered version. ${ }^{32}$ Although previous studies have reported consistent performance between selfadministration and interviewer administration of the PHQ- $9,{ }^{15}$ it is possible that administration mode in this population affected the estimated test characteristics. For example, participants may be less likely to report depressive symptoms to an interviewer than to endorse them on paper, which would be expected to bias the estimate of sensitivity downward. The prevalence of positive screens for dementia of $83 \%$ was higher than in previous research among HIV-infected patients in Cameroon, ${ }^{33}$ suggesting some degree of memory impairment in the study subjects which could also have biased the sensitivity downward in this interviewer administrated version of the PHQ-9. The estimates of test characteristics may also have been influenced by the difference in time frames referenced by the PHQ-9 (past two weeks) and the CIDI (past month), which would also be expected to bias the estimate of sensitivity downward. Our approach, however, was consistent with ${ }^{18}$ or more stringent than ${ }^{19}$ similar studies using the CIDI as the reference standard.

Insufficient evidence was available in this study to distinguish between the competing hypotheses described above for the observed low sensitivity of the PHQ-9. Efforts to identify depression screening protocols with higher sensitivity might focus on (1) conducting validation studies in larger populations or populations with a higher prevalence of depression to permit more precise estimation of sensitivity; (2) testing whether mode of administration affects sensitivity, for example by comparing interviewer administration to audio computer-assisted self-interview (ACASI); and (3) testing whether medical comorbidity, and particularly co-occurrence of dementia, affects sensitivity. Given other reports of low sensitivity of the PHQ-9 in chronically medically ill and non-US populations, ${ }^{19,20,34,35}$ additional research should also directly compare various depression screening instruments to identify which instrument has the best characteristics in a given population.

In conclusion, the PHQ-9 demonstrated high specificity but apparently low sensitivity at the standard cutoff in detecting past-month MDD as measured by the CIDI in this sample of HIV patients receiving ART in an urban center in Cameroon. The high specificity suggests 
that any positive screen will require immediate clinical attention to confirm a diagnosis. On the other hand, the low estimated sensitivity implies a high false negative rate, suggesting that methods to increase opportunities for identifying patients with MDD may be useful. However, sensitivity was extremely imprecisely measured because of the low prevalence of MDD in the sample. Further research may benefit from identifying higher-prevalence populations to improve sensitivity estimates, examining the role of administration mode on PHQ-9 performance, assessing the impact of dementia and other medical comorbidities on test characteristics, and validating alternative depression screening tools. In general, the results of this study underline the importance of formative work such as diagnostic validation studies when applying a depression screening instrument in a new population.

\section{Acknowledgments}

This work was made possible by our study participants and by study personnel Mrs. Shantal Asanji, Dr. Awasum Charles, Mr. Andrew Goodall, Mr. Fru Johnson, Dr. Charles Arrey Kefie, Mrs. Irene Numfor, Mr. Joseph Nyingcho, and Ms. Seema Parkash.

Role of funding source: This study was supported by grant R34 MH084673 of the National Institute of Mental Health, National Institutes of Health, Bethesda, MD, USA. BWP is an investigator with the Implementation Research Institute (IRI), at the George Warren Brown School of Social Work, Washington University in St. Louis; through an award from the National Institute of Mental Health (R25 MH080916-01A2) and the Department of Veterans Affairs, Health Services Research \& Development Service, Quality Enhancement Research Initiative (QUERI). BNG receives funding from the NC TRACS Institute, which is supported by grants UL1RR025747, KL2RR025746, and TLRR025745 from the NIH National Center for Research Resources and the National Center for Advancing Translational Sciences, National Institutes of Health. This publication was made possible with help from the Duke University Center for AIDS Research (CFAR), an NIH funded program (P30 AI064518). The content is solely the responsibility of the authors and does not necessarily represent the official views of the NIMH or the NIH. The funders had no role in study design, data collection and analysis, decision to publish, or preparation of the manuscript.

\section{References}

1. Bing EG, Burnam MA, Longshore D, et al. Psychiatric disorders and drug use among human immunodeficiency virus-infected adults in the United States. Arch Gen Psychiatry. Aug; 2001 58(8):721-728. [PubMed: 11483137]

2. Gonzalez JS, Batchelder AW, Psaros C, Safren SA. Depression and HIV/AIDS Treatment Nonadherence: A Review and Meta-analysis. J Acquir Immune Defic Syndr. Aug 19.2011

3. Leserman J. Role of depression, stress, and trauma in HIV disease progression. Psychosom Med. Jun; 2008 70(5):539-545. [PubMed: 18519880]

4. Rush AJ, Trivedi MH, Ibrahim HM, et al. The 16-Item Quick Inventory of Depressive Symptomatology (QIDS), clinician rating (QIDS-C), and self-report (QIDS-SR): a psychometric evaluation in patients with chronic major depression. Biological psychiatry. Sep 1; 2003 54(5):573583. [PubMed: 12946886]

5. Kaharuza FM, Bunnell R, Moss S, et al. Depression and CD4 cell count among persons with HIV infection in Uganda. AIDS and behavior. Jul; 2006 10(4 Suppl):S105-111. [PubMed: 16802195]

6. Kroenke K, Spitzer RL, Williams JB. The PHQ-9: validity of a brief depression severity measure. J Gen Intern Med. Sep; 2001 16(9):606-613. [PubMed: 11556941]

7. Adewuya AO, Ola BA, Afolabi OO. Validity of the patient health questionnaire (PHQ-9) as a screening tool for depression amongst Nigerian university students. Journal of affective disorders. Nov; 2006 96(1-2):89-93. [PubMed: 16857265]

8. Monahan PO, Shacham E, Reece M, et al. Validity/Reliability of PHQ-9 and PHQ-2 Depression Scales Among Adults Living with HIV/AIDS in Western Kenya. J Gen Intern Med. Nov 20; 2009 24(2):189-197. [PubMed: 19031037]

9. United Nations Development Program. Human development report 2011: Sustainability and equity: A better future for all. UNDP; New York, NY: 2011.

10. UNAIDS. UNAIDS Report on the Global AIDS Epidemic 2010. UNAIDS; Geneva: 2010. 
11. American Psychiatric Association. Diagnostic and Statistical Manual of Mental Disorders. 4th (text revision) ed.. Washington, DC: 2000.

12. Maj M, Janssen R, Starace F, et al. WHO Neuropsychiatric AIDS study, cross-sectional phase I. Study design and psychiatric findings. Arch Gen Psychiatry. Jan; 1994 51(1):39-49. [PubMed: 8279928]

13. Robins LN, Wing J, Wittchen HU, et al. The Composite International Diagnostic Interview. An epidemiologic Instrument suitable for use in conjunction with different diagnostic systems and in different cultures. Arch Gen Psychiatry. Dec; 1988 45(12):1069-1077. [PubMed: 2848472]

14. Demyttenaere K, Bruffaerts R, Posada-Villa J, et al. Prevalence, Severity, and Unmet Need for Treatment of Mental Disorders in the World Health Organization World Mental Health Surveys. JAMA. 2004; 291(21):2581-2590. \%R 10.1001/jama.291.21.2581. June 2, 2004. [PubMed: 15173149]

15. Pinto-Meza A, Serrano-Blanco A, Penarrubia MT, Blanco E, Haro JM. Assessing depression in primary care with the PHQ-9: can it be carried out over the telephone? J Gen Intern Med. Aug; 2005 20(8):738-742. [PubMed: 16050884]

16. Sacktor NC, Wong M, Nakasujja N, et al. The International HIV Dementia Scale: a new rapid screening test for HIV dementia. AIDS (London, England). Sep 2; 2005 19(13):1367-1374.

17. Gilbody S, Richards D, Brealey S, Hewitt C. Screening for depression in medical settings with the Patient Health Questionnaire (PHQ): a diagnostic meta-analysis. J Gen Intern Med. Nov; 2007 22(11):1596-1602. [PubMed: 17874169]

18. Arroll B, Goodyear-Smith F, Crengle S, et al. Validation of PHQ-2 and PHQ-9 to screen for major depression in the primary care population. Ann Fam Med. Jul-Aug;2010 8(4):348-353. [PubMed: 20644190]

19. Zuithoff NP, Vergouwe Y, King M, et al. The Patient Health Questionnaire-9 for detection of major depressive disorder in primary care: consequences of current thresholds in a crosssectional study. BMC family practice. 2010; 11:98. [PubMed: 21144018]

20. McManus D, Pipkin SS, Whooley MA. Screening for depression in patients with coronary heart disease (data from the Heart and Soul Study). The American journal of cardiology. Oct 15; 2005 96(8):1076-1081. [PubMed: 16214441]

21. Nakimuli-Mpungu E, Bass JK, Alexandre P, et al. Depression, Alcohol Use and Adherence to Antiretroviral Therapy in Sub-Saharan Africa: A Systematic Review. AIDS and behavior. Nov 25.2011

22. Adewuya AO, Afolabi MO, Ola BA, et al. Relationship between depression and quality of life in persons with HIV infection in Nigeria. Int J Psychiatry Med. 2008; 38(1):43-51. [PubMed: 18624016]

23. Lawler K, Mosepele M, Ratcliffe S, et al. Neurocognitive impairment among HIV-positive individuals in Botswana: a pilot study. Journal of the International AIDS Society. 2010; 13:15. [PubMed: 20406460]

24. Marwick KF, Kaaya SF. Prevalence of depression and anxiety disorders in HIV-positive outpatients in rural Tanzania. AIDS Care. Apr; 2010 22(4):415-419. [PubMed: 20131127]

25. Myer L, Smit J, Roux LL, Parker S, Stein DJ, Seedat S. Common mental disorders among HIVinfected individuals in South Africa: prevalence, predictors, and validation of brief psychiatric rating scales. AIDS patient care and STDs. Feb; 2008 22(2):147-158. [PubMed: 18260806]

26. Olley BO, Seedat S, Stein DJ. Persistence of psychiatric disorders in a cohort of HIV/AIDS patients in South Africa: a 6-month follow-up study. Journal of psychosomatic research. Oct; 2006 61(4):479-484. [PubMed: 17011355]

27. Spies G, Kader K, Kidd M, et al. Validity of the K-10 in detecting DSM-IV-defined depression and anxiety disorders among HIV-infected individuals. AIDS Care. Sep; 2009 21(9):1163-1168. [PubMed: 20024776]

28. Kinyanda E, Hoskins S, Nakku J, Nawaz S, Patel V. Prevalence and risk factors of major depressive disorder in HIV/AIDS as seen in semi-urban Entebbe district, Uganda. BMC psychiatry. 2011; 11:205. [PubMed: 22208452]

29. Sheehan DV, Lecrubier Y, Sheehan KH, et al. The Mini-International Neuropsychiatric Interview (M.I.N.I.): the development and validation of a structured diagnostic psychiatric interview for 
DSM-IV and ICD-10. J Clin Psychiatry. 1998; 59(Suppl 20):22-33. quiz 34-57. [PubMed: 9881538]

30. Omoro SA, Fann JR, Weymuller EA, Macharia IM, Yueh B. Swahili translation and validation of the Patient Health Questionnaire-9 depression scale in the Kenyan head and neck cancer patient population. Int J Psychiatry Med. 2006; 36(3):367-381. [PubMed: 17236703]

31. Okulate GT, Olayinka MO, Jones OB. Somatic symptoms in depression: evaluation of their diagnostic weight in an African setting. Br J Psychiatry. May.2004 184:422-427. [PubMed: 15123506]

32. Esler D, Johnston F, Thomas D, Davis B. The validity of a depression screening tool modified for use with Aboriginal and Torres Strait Islander people. Australian and New Zealand journal of public health. Aug; 2008 32(4):317-321. [PubMed: 18782392]

33. Njamnshi AK, Djientcheu Vde P, Fonsah JY, Yepnjio FN, Njamnshi DM, Muna WE. The International HIV Dementia Scale is a useful screening tool for HIV-associated dementia/ cognitive impairment in HIV-infected adults in Yaounde-Cameroon. J Acquir Immune Defic Syndr. Dec 1; 2008 49(4):393-397. [PubMed: 19186351]

34. Persoons P, Luyckx K, Desloovere C, Vandenberghe J, Fischler B. Anxiety and mood disorders in otorhinolaryngology outpatients presenting with dizziness: validation of the self-administered PRIME-MD Patient Health Questionnaire and epidemiology. Gen Hosp Psychiatry. Sep-Oct;2003 25(5):316-323. [PubMed: 12972222]

35. Picardi A, Adler DA, Abeni D, et al. Screening for depressive disorders in patients with skin diseases: a comparison of three screeners. Acta dermato-venereologica. 2005; 85(5):414-419. [PubMed: 16159733] 


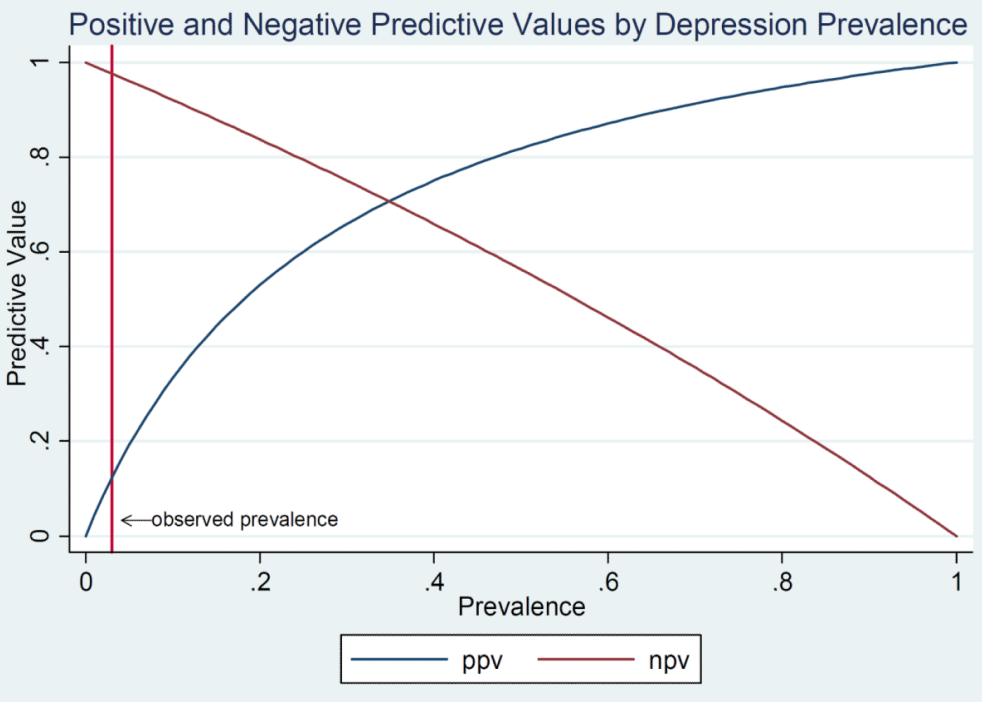

Figure 1. Positive and negative predictive values of PHQ-9 by depression prevalence PPV: Positive predictive value. NPV: Negative predictive value. 


\section{Table 1}

Characteristics of the overall study population $(n=400)$

\begin{tabular}{|c|c|}
\hline Characteristic & $\mathrm{n}(\%)$ or median (IQR) \\
\hline \multicolumn{2}{|l|}{ Sex } \\
\hline Male & $103(26)$ \\
\hline Female & $297(74)$ \\
\hline Age & $41(34-47)$ \\
\hline \multicolumn{2}{|l|}{ Religion } \\
\hline Christian & $394(99)$ \\
\hline Other & $5(1)$ \\
\hline \multicolumn{2}{|l|}{ Marital status } \\
\hline Married/cohabitating & $137(34)$ \\
\hline Previously married & $178(44)$ \\
\hline Never married & $85(21)$ \\
\hline \multicolumn{2}{|l|}{ Education $*$} \\
\hline Primary & $245(61)$ \\
\hline Greater than primary & $155(39)$ \\
\hline Daily expenditures ${ }^{* *}$ & US\$1 (\$1-3) \\
\hline \multicolumn{2}{|l|}{ Village of residence } \\
\hline Urban & $244(61)$ \\
\hline Rural & $156(39)$ \\
\hline HIV symptom score (possible range: $0-13$ ) & $5(3-6)$ \\
\hline Dementia score (possible range: $4-12$ ) & $9(8-10)$ \\
\hline Negative screen $(>10)$ & $66(17)$ \\
\hline Positive screen $(\leq 0)$ & $333(83)$ \\
\hline \multicolumn{2}{|c|}{ Primary $=6$ years or fewer; greater than primary=more than 6 years } \\
\hline \multicolumn{2}{|c|}{ In US dollars, approximation based on reported weekly expenditures in FCFA (Central Africa CFA francs). } \\
\hline
\end{tabular}




\section{Table 2}

Comparison of PHQ-9 screening results to depressive diagnosis using CIDI

\begin{tabular}{ccc}
\hline & \multicolumn{2}{c}{ MDD, past month } \\
\cline { 2 - 3 } PHQ-9 score & Depression & No depression \\
\hline$\geq 10$ & 3 & 23 \\
$<10$ & 8 & 364 \\
Total & 11 & 387 \\
\hline
\end{tabular}

CIDI: Composite International Diagnostic Interview. MDD: Major Depressive Disorder. 


\section{Table 3}

PHQ-9 screening characteristics at selected cut-off values

\begin{tabular}{lllll}
\hline Cut-point & Sensitivity (95\% CI) & Specificity (95\% CI) & LR+ & LR- \\
\hline PHQ-9 $\geq 8$ & $0.36(0.11,0.69)$ & $0.89(0.86,0.92)$ & 3.3 & 0.7 \\
PHQ-9 $\geq 10$ & $0.27(0.06,0.61)$ & $0.94(0.91,0.96)$ & 4.5 & 0.8 \\
PHQ-9 $\geq 12$ & $0.18(0.02,0.52)$ & $0.97(0.95,0.98)$ & 6.0 & 0.8 \\
\hline
\end{tabular}

LR: Likelihood ratio. 
Table 4

Comparison of specificity of PHQ-9 screening between subgroups

\begin{tabular}{llcc}
\hline Characteristic & Prevalence of past-month depression (CIDI) & Specificity of PHQ-9 & Exact P-value (two-tailed) \\
\hline Gender & $4 \%$ & & \\
$\quad$ Male & $2 \%$ & $0.94(0.87,0.98)$ & \\
$\quad$ Female & & $0.94(0.91,0.97)$ & 1.00 \\
HIV symptom score & $2 \%$ & & \\
$\quad \mathbf{5}$ & $4 \%$ & $0.96(0.92,0.98)$ & 0.14 \\
$\quad \mathbf{5}$ & & $0.92(0.88,0.96)$ & \\
IHDS result & $2 \%$ & & \\
$\quad$ Positive dementia screen & $5 \%$ & $0.95(0.92,0.97)$ & 0.24 \\
$\quad$ Negative dementia screen & $5 \%(0.80,0.96)$ & \\
\hline
\end{tabular}

CIDI: Composite International Diagnostic Interview. IHDS: International HIV Dementia Scale 